\title{
Colour filtering in a-SiC:H based p-i-n-p-i-n cells: A trade-off between bias polarity and absorption regions
}

\author{
M. Vieira ${ }^{\text {a,* }}$, A. Fantoni ${ }^{\text {a }}$, P. Louro ${ }^{\text {a }}$, M. Fernandes ${ }^{\text {a }}$, J. Martins $^{\text {a }}$, \\ R. Schwarz ${ }^{\mathrm{a}}$, G. Lavareda ${ }^{\mathrm{b}}$, C.N. Carvalho ${ }^{\mathrm{b}}$ \\ ${ }^{a}$ Electronics Telecommunication and Computer Department ISEL, R. Conselheiro Emídio Navarro, 1949-014 Lisboa, Portugal \\ ${ }^{\mathrm{b}}$ DCM, FCT-UNL, Quinta da Torre, 2829-516 Caparica, Portugal
}

Received 9 September 2005; received in revised form 2 February 2006; accepted 3 February 2006

Available online 29 March 2006

\begin{abstract}
A large area colour imager optically addressed is presented. The colour imager consists of a thin wide band gap p-i-n a-SiC:H filtering element deposited on the top of a thick large area a-SiC:H(-p)/a-Si:H(-i)/a-SiC:H(-n) image sensor, which reveals itself an intrinsic colour filter.

In order to tune the external applied voltage for full colour discrimination the photocurrent generated by a modulated red light is measured under different optical and electrical bias. Results reveal that the integrated device behaves itself as an imager and a filter giving information not only on the position where the optical image is absorbed but also on it wavelength and intensity.

The amplitude and sign of the image signals are electrically tuneable. In a wide range of incident fluxes and under reverse bias, the red and blue image signals are opposite in sign and the green signal is suppressed allowing blue and red colour recognition. The green information is obtained under forward bias, where the blue signal goes down to zero and the red and green remain constant. Combining the information obtained at this two applied voltages a RGB colour image picture can be acquired without the need of the usual colour filters or pixel architecture. A numerical simulation supports the colour filter analysis.
\end{abstract}

(C) 2006 Elsevier B.V. All rights reserved.

Keywords: Colour and image sensitive detectors; Spectral sensitivity; Light filtering; Self-biasing effect; Multi-layer devices

\section{Introduction}

Red, green and blue (RGB) are the additive fundamental perceptual components of the visible spectrum and can be easily separated by using appropriate detectors and/or bandpass filters $[1,2]$. Light filtering, employing distinct wavelength optimized structures, in an array, is rather complex and is an expensive solution. Optical filters may be eliminated by using a-SiC:H multi-layer stacked devices, in which the detector structure, itself, behaves as a filter. In the stacked structure, information about the spectrum corresponds to information about where the radiation is absorbed. By sampling the absorption region with different bias voltages, and extracting separately the integrated information about the radiation absorbed in each region is possible to identify the RGB components of the visible spectrum

\footnotetext{
* Corresponding author. Tel.: +35121 8317290; fax: +351218317114.

E-mail address: mv@isel.ipl.pt (M. Vieira).
}

[3-6]. In those devices, blue light is usually detected in the front of the structure, green light at the centre and red light at the bottom.

Large area single and stacked p-i-n image sensors based on amorphous hydrogenated silicon alloys were proposed as optically addressed laser scanned photodiode (LSP) $[7,8]$. The LSP sensor is different from the electrically scanned image systems since it is based on one single sensing element, and uses a modulated low-power beam of laser light to scan the active area directly. Advantages to this approach are large area imaging, high resolution and uniformity of measurement along the sensor. The complexity of the interconnection is reduced, while the colour information is extracted by applying a sequence of test voltages.

In this paper, we propose to optimize the sensor design in order to apply the optical addressed Laser Scanned Photodiode technique to full colour discrimination. The effect of the applied voltage on the colour selectivity and image intensity is discussed and supported by a self-biasing model. 


\section{Sensor design, characterization and operation}

The sensing element consists of two stacked amorphous cells $\quad[p(\mathrm{SiC}: \mathrm{H}) / \mathrm{i}(\mathrm{SiC}: \mathrm{H}) / \mathrm{n}(\mathrm{SiC}: \mathrm{H}) / \mathrm{p}(\mathrm{SiC}: \mathrm{H}) / \mathrm{i}(\mathrm{Si}: \mathrm{H}) / \mathrm{n}(\mathrm{Si}: \mathrm{H})]$ and two conductive contacts. As transparent conductors are required, for both front and back illuminated surfaces, indium tin oxide, produced by thermal evaporation, was used. The intrinsic and doped layers were fabricated by plasma enhanced chemical vapour deposition at $13.56 \mathrm{MHz}$ radio frequency.

The design attempts to optimize the most important performance attributes of the colour imager which are the spatial resolution, the colour sensitivity and the dynamic range. In this configuration, full colour detection is attempted based on spatially separated absorption of different wavelengths. The blue sensitivity and the red transmittance were optimized, respectively, through a thin a-SiC:H absorber $(200 \mathrm{~nm})$ with an optical gap of $2.1 \mathrm{eV}$ and thick a-SiH back absorbers $(\mathrm{NC} \# 4 / 500 \mathrm{~nm}$; $\mathrm{NC} \# 5 / 1000 \mathrm{~nm}$ ) having optical gap around $1.8 \mathrm{eV}$. Their thicknesses are a trade-off between the full absorption of the blue light into the front diode and green across both. To decrease the lateral currents that could lead to image smearing and to enhance the blue sensitivity, the doped layers $(20 \mathrm{~nm}$ thick) are based on a-SiC:H [9]. The doped layers provide rectifying contacts but do not contribute to the light sensitivity because doping causes a high density of charge dangling bond defects in a-SiC:H. Doping level in the internal recombination junction is about half the doping level of the external $\mathrm{p}$ and $\mathrm{n}$ layers. The internal lightly doped layers are ionized and cause a reduction of the field at the contacts increasing the breakdown field.

The image to acquire is optically mapped onto the front photodiode and a low-power light spot $\left(\lambda_{\mathrm{S}}=650 \mathrm{~nm}\right.$; $\Phi_{\mathrm{S}}=10 \mu \mathrm{W} \mathrm{cm}{ }^{-2}$ ) scans the device by the opposite side. The photocurrent generated by the moving spot is recorded as the electronic image signal, and its magnitude depends on the light pattern localization, wavelength and intensity [3]. The line scan speed is close to $1 \mathrm{kHz}$. No image processing algorithms are used during the image reconstruction process.

The devices were characterized through the analysis of the photocurrent and spectral response (in the range of $400-800 \mathrm{~nm}$ ) under different RGB optical bias $\left(\lambda_{\mathrm{L}}=450,550\right.$ and $650 \mathrm{~nm}$; $\left.\Phi_{\mathrm{L}}=200 \mu \mathrm{W} \mathrm{cm}^{-2}\right)$ and in dark, for different applied voltages $(-6 \mathrm{~V}<V<6 \mathrm{~V})$. In these measurements the device was uniformly illuminated through the a-Si:H back diode with red chopped light $\left(\lambda_{\mathrm{S}}=650 \mathrm{~nm}, \Phi_{\mathrm{S}}=10 \mu \mathrm{W} \mathrm{cm}{ }^{-2}\right)$ and the optical bias was applied through the a-SiC:H front one. The photocurrent under different optical and electric bias conditions was measured using a lock-in amplifier.

\section{Experimental results}

\subsection{Sensor responsivity and colour rejection}

In order to optimize, for each sensor, the applied voltages that lead to colour filtering and to evaluate the responsivity to different light pattern wavelengths, the photocurrent generated
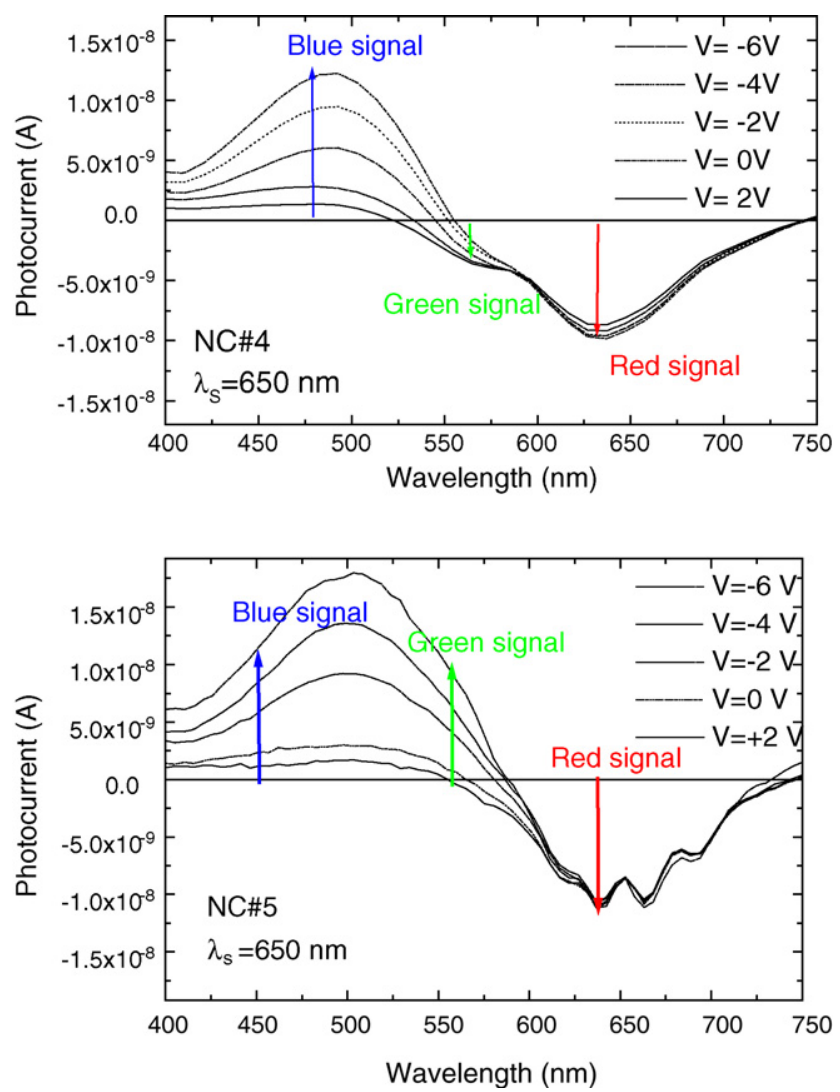

Fig. 1. Image signal as a function of the optical bias and under different applied voltages.

by a red pulsed laser (the scanner) was measured under different steady-state illumination conditions and different electrical bias. This image signal (difference between the photocurrent with and without optical bias) is displayed in Fig. 1. Here the photocurrent at $750 \mathrm{~nm}$ was assumed as the dark level.

In order to tune correctly, for each sensor, the readout voltage that enables colour rejection (no image signal), the photocurrents generated by the same scanner, as a function of the applied bias and under different steady-state illumination conditions, are displayed in Fig. 2 and compared with their values without optical bias. The arrows (dotted in sensor $\mathrm{NC} \# 4$ and solid in $\mathrm{NC}$ \#5) guide the eyes towards the values where colour rejection is achieved (crossover between the photocurrents with and without optical bias).

To be sure that these readout voltages are independent on the image intensity, the photocurrent generated by the scanner was measured under red, green and blue steady-state illumination $\left(0 \mu \mathrm{W} \mathrm{cm} \mathrm{cm}^{-2}<\Phi_{\mathrm{L}}<160 \mu \mathrm{W} \mathrm{cm}^{-2}\right)$. In these measurements the element sensor was uniformly illuminated through the front diode with red pulsed light and the optical bias applied through the back one. For sensor NC \#5, Fig. 3 shows this dependence.

\subsection{Image and colour recognition}

To show the ability of the sensor as a colour image sensor, the acquired images (difference between the photocurrents with and 


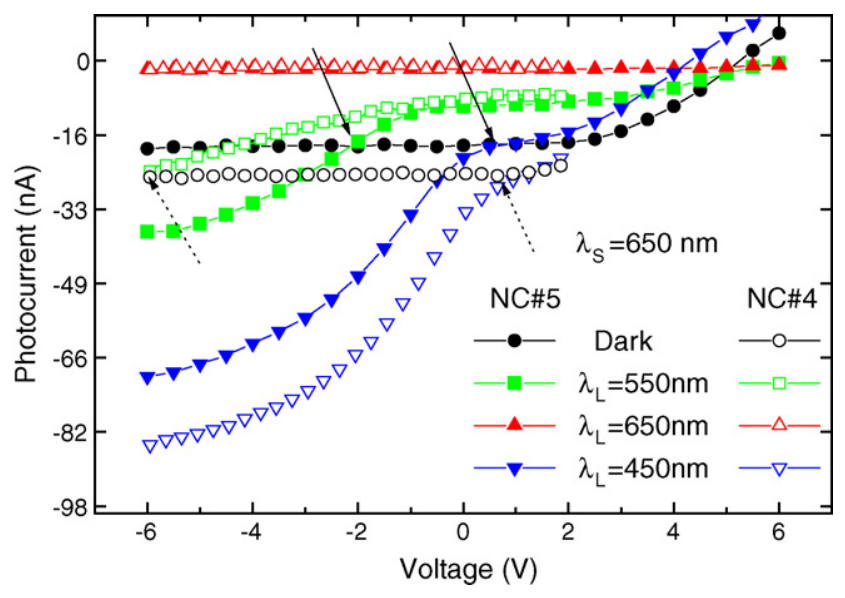

Fig. 2. Photocurrent as a function of the applied bias for sensors NC \#4 (a) and NC \#5 (b) in dark and under blue, green and red irradiation. (For interpretation of the references to colour in this figure legend, the reader is referred to the web version of the article.)

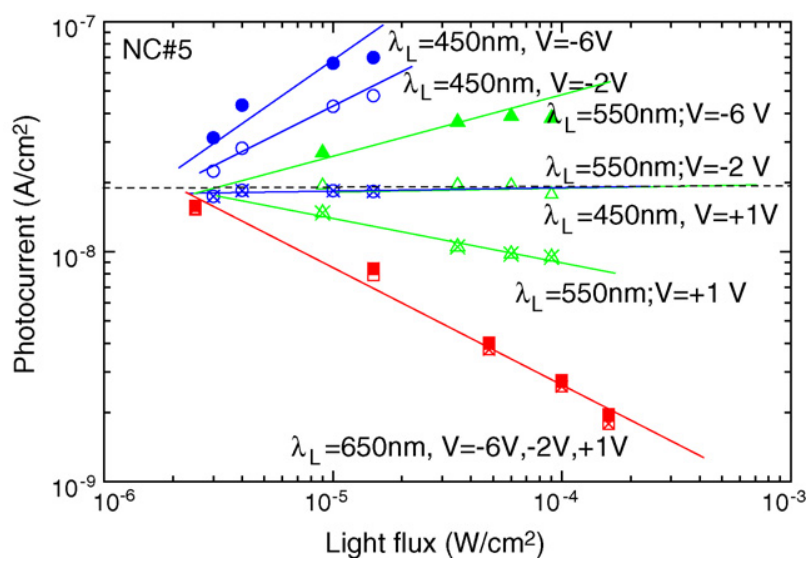

Fig. 3. Photocurrent as a function of the incident flux.

without a light pattern) are shown in Fig. 4 for sensor NC \#5, at $-6,-2$ and $+1 \mathrm{~V}$. Here, the same green, red and blue optical images (5) were projected, one by one, on the active surface of the front diode and acquired through the back one with the LSP technique. The line scan frequency was close to $1 \mathrm{kHz}$ and no algorithms were used during the image restoration process.

\begin{tabular}{|l|l|l|l|}
\hline & $-6 \mathrm{~V}$ & $-2 \mathrm{~V}$ & $+1 \mathrm{~V}$ \\
\hline Red & 5 & -5 & \\
\hline Green & - & & \\
\hline Blue & & & \\
\hline
\end{tabular}

Fig. 4. Red $\left(\lambda_{\mathrm{L}}=650 \mathrm{~nm}\right)$, green $\left(\lambda_{\mathrm{L}}=550 \mathrm{~nm}\right)$ and blue $\left(\lambda_{\mathrm{L}}=450 \mathrm{~nm}\right)$ electrical images acquired by the LSP technique, at different applied bias. (For interpretation of the references to colour in this figure legend, the reader is referred to the web version of the article.)
For readout time of $1 \mathrm{~ms}$ the frame time, for a 50 lines image, is around $50 \mathrm{~ms}$. The white cross guide the eyes for the colour rejection at each voltage.

\section{Simulation results}

We used a device simulation program ASCA-2D [10] to analyze the potential profiles in the investigated structure. The thickness of the front and back diodes and the optical gaps were chosen in compliance with the obtained experimental values. The doping level was adjusted in order to obtain approximately the same conductivity of the layers as in the tested samples. The photogeneration/recombination profiles for a NC \#5-like cell are depicted in Fig. 5, under red, green and blue irradiation.

In Fig. 6, the obtained potential profiles are compared in dark and under red, green and blue irradiation, at $-4,-2$ and $+1 \mathrm{~V}$.

Fig. 7 displays the simulated electrical field profiles at different electrical bias, under blue (a), green (b), red (c) irradiation and in dark (d).
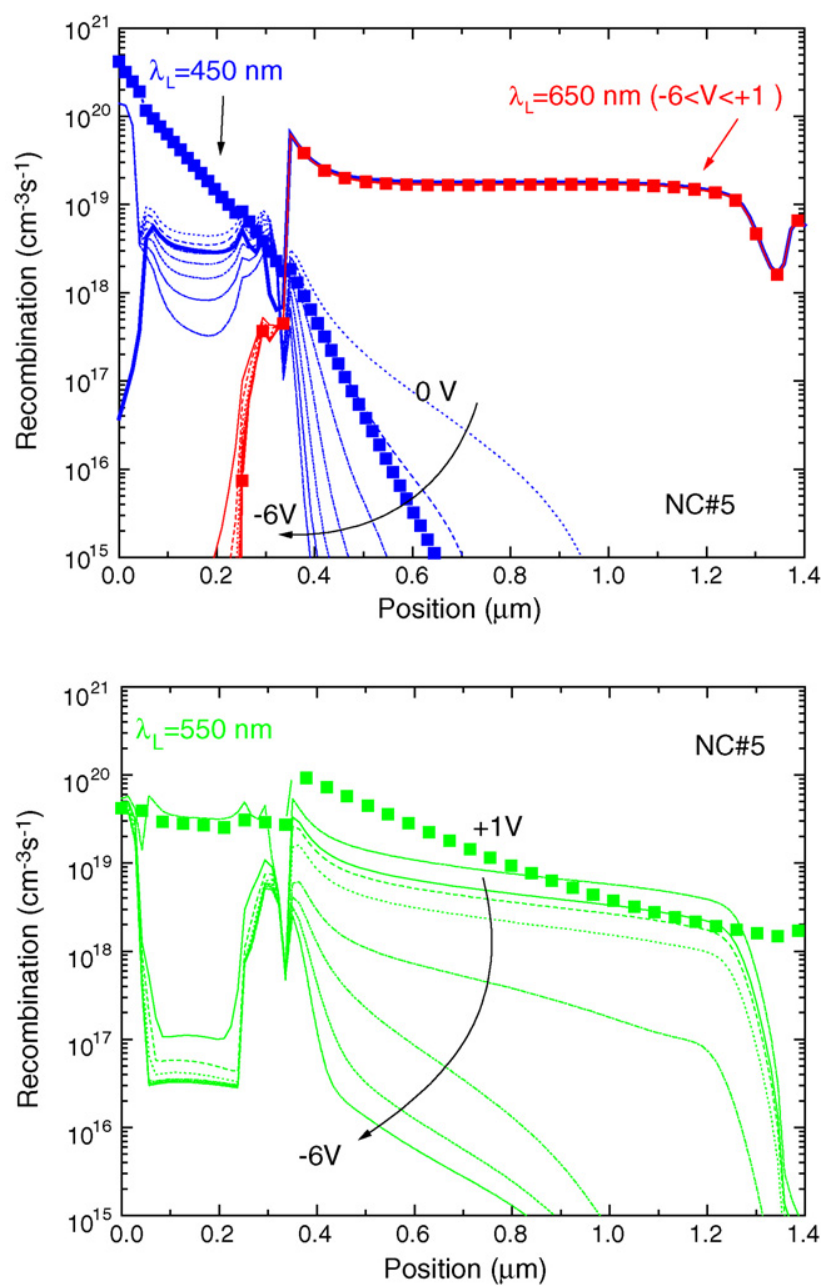

Fig. 5. Recombination profiles (straight lines) under red $\left(\lambda_{\mathrm{L}}=650 \mathrm{~nm}\right)$ green $\left(\lambda_{\mathrm{L}}=550 \mathrm{~nm}\right)$ and blue $\left(\lambda_{\mathrm{L}}=450 \mathrm{~nm}\right)$ optical bias and different electrical bias. The generation profiles are also shown (symbols). (For interpretation of the references to colour in this figure legend, the reader is referred to the web version of the article.) 


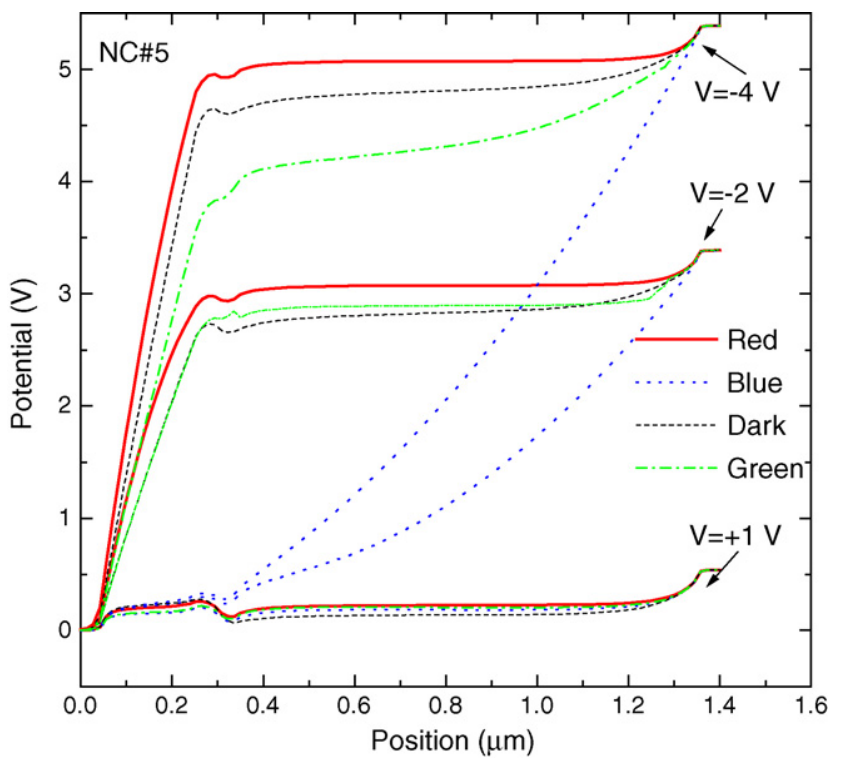

Fig. 6. Potential profiles at different electrical bias for sensor NC \#5 under blue, green, red irradiation and without optical bias. (For interpretation of the references to colour in this figure legend, the reader is referred to the web version of the article.)

\section{Discussion}

\subsection{Colour filtering}

Results show that the light-to-dark responsivity to a red pulsed light (Figs. 1 and 2) depends on thickness of the back diode and on the applied voltage. As the applied bias changes from forward to reverse, in both sensors, the responsivity increases in the blue range and remains constant in the red one. The main difference occurs in the green spectral region. In the thin device ( $\mathrm{NC} \mathrm{\# 4)} \mathrm{the} \mathrm{behaviour} \mathrm{is} \mathrm{similar} \mathrm{to} \mathrm{the} \mathrm{one}$ observed under red illumination while in the thick one (NC \#5) it approaches the trend under blue irradiation.

Under red irradiation or in dark (without optical bias) the photocurrent generated by the scanner is independent on the applied voltage (Fig. 2). This effect can be ascribed to the flatness of the potential across the back absorber in a wide range of applied voltages (Fig. 6). Under blue/green irradiation the responsivity decreases as the applied voltage changes from reverse to forward due to an asymmetric decrease of the electrical field across front and back absorbers (Fig. 7) until the flat band condition is achieved. It is interesting to notice that around $-6 \mathrm{~V}$ (NC \#4) or $-2 \mathrm{~V}(\mathrm{NC} \mathrm{\# 5)}$ the collection with or without green image is the same, leading to the rejection of the green image signal

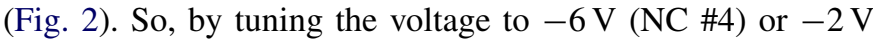
(NC \#5) the red and blue signals are high and opposite in sign and the green signal is suppressed allowing blue and red colour recognitions. The green information is obtained under slight forward bias $(+1 \mathrm{~V})$, where the blue signal goes down to zero and the red remains constant. Fig. 3 also shows that there is a good reproducibility between the flux intensity and the amplitude of the photocurrent, which reinforces the suppression of the blue
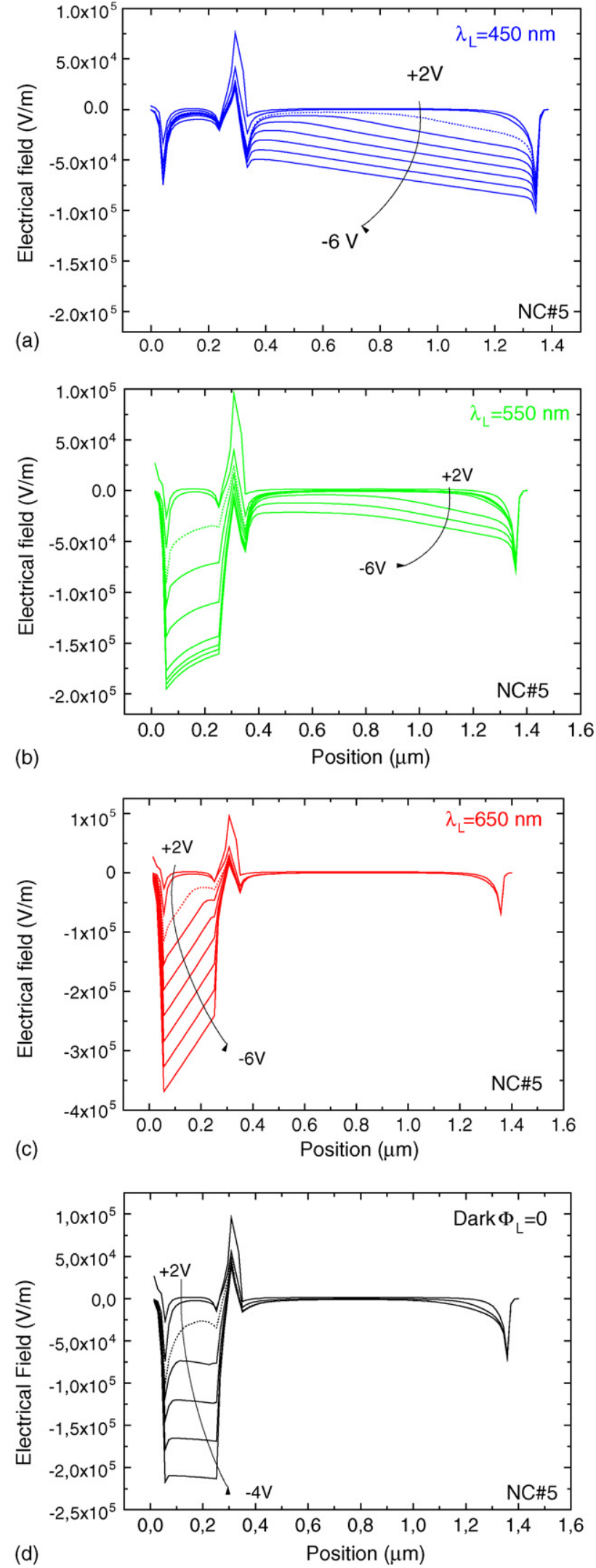

Fig. 7. Simulated electrical field profile under different applied voltages, under blue (a) green (b) and red (c) irradiations and without optical bias (d). (For interpretation of the references to colour in this figure legend, the reader is referred to the web version of the article.) 
and green signals at +1 and $-2 \mathrm{~V}$, respectively, independently of the light flux of the optical bias. Combining the information obtained at three applied voltages a full colour image can be acquired without the need of the usual colour filters or pixel architecture as can be seen in Fig. 4.

\subsection{Self-biasing effect}

Figs. 5-7 show that the shallow penetration of the blue photons into the front diode, the deep penetration of the red photons exclusively into the back diode or the decay of the green absorption across both controls the sensor behaviour. In the analyzed structures both the front and the back diodes are optically and electrically in series. Since light traverses through the sequence and is absorbed accordingly its wavelength (Fig. 5) and since they are electrically in series, the current through both diodes should be the same. Any diode whose current is bellow the other has to reduce its bucking current and consequently voltage to try to catch up. This diode may even have to reverse bias itself in its efforts to get in line with the other. This effect is what we call the self-biasing effect.

Results show that the application of an external electrical bias (forward or reverse) mainly influences the field distribution within the less photo-excited sub-cell (Figs. 5 and 7). When compared with the electric field profile under thermo-dynamical equilibrium conditions, the field under illumination conditions is lowered in the most absorbing cell, while the less absorbing one reacts by assuming a reverse bias configuration. Hence, opposite behaviour is observed under red and blue background light while under green light condition the redistribution of the field profile is balanced between the two sub-cells. The balance between those electrical field adjustments, due to the non-uniform absorption of the impinging photons, depends on generation/recombination ratio profiles at each applied voltage. As this ratio approaches the unity the self-bias effect changes from reverse to forward until flat band condition is achieved. To sustain the net current across the device, under blue irradiation, the current in the back diode has to be adjusted through an increase of the electrical field that becomes reverse bias even under short circuit conditions (Fig. 7). As the voltage changes from reverse to forward the electrical field decreases from the bottom to the front side of the back diode and approaches its value in dark around $+1 \mathrm{~V}$, in both sensors, leading to colour rejection (arrows in Fig. 2). The deep penetration of the red photons uniformly and exclusively into the switching diode, and the transparency of the sensing diode to them push the electrical field up in the front diode (self-reverse effect) and down into the back one (self-forward effect) leading to a poor collection of the carriers generated by the red scanner (Fig. 2). In the green range both reverse and forward self-bias effects co-exists. The observed differences are related with the decay of the green absorption across both sensors (Fig. 5). Here, the self-reverse effect due to the front absorption is balanced by the self-forward effect due to the back absorption, that is higher inside the thin device. So, to cancel this effect a higher reverse bias $(-6 \mathrm{~V})$ is needed in the thin device then in the thicker one $(-2 \mathrm{~V})$.

\section{Conclusions}

Light filtering using the Laser Scanned Photodiode technique in a-SiC:H multi-layer devices was presented and explained through a trade-off between the different light penetration depth of the impinging photons and self adjustments of the electrical field.

Results show that the colour discrimination and the lightto-dark sensitivity are dependent on the thickness of the back absorber and on the readout voltage. For each red, green and blue wavelength colour rejection was achieved at a readout voltage that cancels the self-bias effect in the back absorber. This voltage shifts from reverse to forward as the light penetration depth in the back diode decreases.

\section{Acknowledgement}

This work has been financially supported by project POCTI/ESE/38689/2001.

\section{References}

[1] C.P. Sanbdbank (Ed.), Digital Television, John Wiley \& Sons, 1990.

[2] G. de Graaf, F.R. Riedijk, R.F. Wolffenbuttel, Colour sensor system with a frequency output and an ISS or I2C bus interface, Sens. Actuators A61 (1997) 441-445.

[3] H.K. Tsai, S.C. Lee, An amorphous SiC/Si two-color detector, IEEE Electron Device Lett. EDL-8 (1987) 365-367.

[4] H. Stiebig, J. Gield, D. Knipp, P. Rieve, M. Bohm, Amorphous silicon three color detector, in: Amorphous Silicon Technology, vol. 337, 1995, pp. 815-821.

[5] F. Palma, in: R.A. Street (Ed.), Technology and Applications of Amorphous Silicon, Spring-Verlag, Berlin/Heidelberg/New York, 2000.

[6] M. Vieira, M. Fernandes, A. Fantoni, P. Louro, R. Schwarz, A new CLSP sensor for Image recognition and color separation, in: Abelson Cohen, Robertson Matsumura (Eds.), Amorphous and Heterogeneous SiliconBased Films-2002, vol. 715, 2002, pp. 695-700.

[7] M. Vieira, M. Fernandes, P. Louro, R. Schwarz, M. Schubert, Image capture devices based on p-i-n silicon carbides for biometrics applications, J. Non Cryst. Solids 299-302 (2002) 1245-1249.

[8] M. Vieira, A. Fantoni, M. Fernandes, P. Louro, I. Rodrigues, Stacked n-i-p$\mathrm{n}$-i-p heterojunctions for image recognition, in: Amorphous and Nanocrystalline Silicon-Based Films-2003, vol. 762, 2003, p. A.18.13.

[9] M. Vieira, M. Fernandes, P. Louro, A. Fantoni, R. Schwarz, Optically addressed read-write device based on a tandem heterostructure, J. Non Cryst. Solids 338-340 (2004) 754-757.

[10] A. Fantoni, M. Vieira, R. Martins, Simulation of hydrogenated amorphous and microcrystalline silicon optoelectronic devices, Math. Comput. Simul. 49 (1999) 381-401.

\section{Biographies}

M. Vieira was born in Lisbon, Portugal. In 1986, she received the masters of science in solid state physics-microelectronic and in 1993 the $\mathrm{PhD}$ in semiconductor materials both from the New University of Lisbon. She is full professor in electronics inside the department of Electronics Telecommunication and Computers (ISEL, Portugal) and the head of a Group in Applied Research in Microelectronic Optoelectronic and Sensors-GIAMOS. She has several scientific papers and 20 years of experience in the field of thin films and devices, her research activities have been mainly related to the development of optical sensors.

A. Fantoni was born in Rome (Italy) in 1966. He received a university degree in applied mathematics from the University of Camerino, Italy (1992), 
and a $\mathrm{PhD}$ in material engineering/micro and optoelectronics from the New University of Lisbon, Portugal (1999). He actually teaches semiconductor physics in the Electronics, Telecommunications and Computer Department on the Engineering Institute of Lisbon. His research interests are related to numerical analysis and simulation of micro and optoelectronic thin film devices.

P. Louro was born in Portugal in 1967. In 1990, she became researcher in EID Lisbon, Portugal, in the department of optoelectronics. She graduated in physics by the Faculty of Sciences from the University of Lisbon in 1990. In 1995, she received the master of science in material engineering by the New University of Lisbon. Currently, she is assistant professor in the electronics department of ISEL, Lisbon, Portugal, where she teaches electronic and semiconductor physics. Her main research interest is in the field of amorphous semiconductor thin films and devices.

M. Fernandes was born in Portugal in 1970. He graduated in physics and materials engineering by the Faculty of Sciences and Technology from the New University of Lisbon in 1995. Currently, he is assistant professor in Electronics Department of ISEL, Lisbon, Portugal and investigator in the Group of Applied Research in Microelectronic Optoelectronic and Sensors-GIAMOS in the same institution.
J. Martins was born in Lisbon, Portugal. He got his MSc degree in Electronic and Computer from Instituto Superior Técnico in Lisbon and is a assistant professor in the Electronic Telecomunication and Computer Departement of the Instituto Superior de Engenharia de Lisboa. His main scientific interests are: semiconductor devices with emphasis in electrical and numerical simulations.

R. Schwarz was born in Germany. He was a researcher in Physic in Technische Universität München and actually he is a full professor in Instituto Superior Técnico, Lisbon. His main scientific interests are: transport properties in semiconductor materials and their application in devices.

G. Lavareda was born in Lisbon, Portugal. He got his $\mathrm{PhD}$ degree in Materials Engineering from New University of Lisbon and is a full professor in the Materials Science Department of this university. His main scientific interests are: semiconductor materials and devices with emphasis in new transparent TFT structures.

C. Nunes de Carvalho was born in São Paulo, Brasil. He has a PhD in Materials Science from New University of Lisbon and is a full professor in the Materials Science Department of this university. His main scientific interests are: semiconductor and transparent/conductive materials and their application in devices. 